\title{
A Sphere-Packing Modulated Space-Frequency Diversity Aided FFH-Assisted DSTBC System
}

\author{
N. Wu, S. Ahmed, O. Alamri, L.L. Yang and L. Hanzo \\ School of ECS, University of Southampton, SO17 1BJ, UK. \\ Email: \{nw04r, sa03r, ora02r, lly, lh\}@ecs.soton.ac.uk; http://www-mobile.ecs.soton.ac.uk
}

\begin{abstract}
In this treatise we demonstrate, how a high spatial and frequency diversity can be achieved by combining Differential Space-Time Block Coding (DSTBC) and Fast Frequency Hopping (FFH). The proposed FFH assisted DSTBC scheme is capable of achieving a high diversity order given by the product of the frequency diversity order achieved by FFH and by the spatial diversity order offered by the DSTBC arrangement. Hence, the system is capable of exploiting the advantages of both DSTBC and FFH. Furthermore it is less vulnerable to some of their individual disadvantages, such as the performance degradation of DSTBC due to fast fading and the large bandwidth requirement of the FFH system. The proposed system also benefits from the employment of sphere packing and iterative turbo detection.
\end{abstract}

\section{INTRODUCTION}

The family of coherently detected space-time block codes [1] [2] attains full spatial diversity, provided that the nondispersive channel's envelope can be sufficiently accurately estimated at the receiver. Hence, in Multiple Input Multiple Output (MIMO) systems, training symbols have to be transmitted from all the transmit antennas. However, since the total transmit energy is shared by the multiple antennas, the energy available for training symbols is reduced compared to a single antenna aided system, which typically results in an increased estimation error. Furthermore, when the mobile travels at a high speed, the channel's complex envelope varies dramatically, thus accurate channel estimation is a challenging task. Therefore, differentially encoded schemes, invoking noncoherent detection have been proposed in [3] [4] [5]. In this contribution we investigate Differential Space-time Block Coding (DSTBC) based on Phase Shift Keying (PSK), which benefits from an orthogonal design and from a low detection complexity. The PSK-based design of [3] was extended to QAM constellations in [6]. However, an increased diversity order can only be achieved by employing an increased number of transmit antennas, which results in an increased complexity. Furthermore, although orthogonal transmit diversity schemes are capable of attaining the maximum achievable diversity gain, they fail to achieve the maximum possible multiplexing gain.

The financial support of the EPSRC, UK and Euroepan Union under the auspices of the Phoenix and Newcom projects and of the Higher Education Commission, Pakistan is gratefully acknowledged.
In contrast to the above-mentioned spatial diversity schemes, Fast Frequency Hopping (FFH) schemes aim for achieving frequency diversity by hopping to independently faded carriers during a single transmitted symbol duration. However, in practice the statistical behaviour of the mobile channel obeys natural laws, which are beyond the mobile radio system designer's control and hence it cannot always be ensured that the spatial or frequency diversity channels fade independently. Furthermore, the achievable spatial or frequency diversity order is limited by the number of transmit antennas and the number of FFH chips determined by the total bandwidth available, respectively. Therefore, it is beneficial to design a combined DSTBC and FFH aided system, which is potentially capable of combining the achievable spatial and frequency diversity. This specific system configuration is particularly attractive, since FFH systems are non-coherent by nature [7], prohibiting the estimation of channel state information during each hop duration, since the hop duration may be extremely low compared to the symbol duration. Therefore, coherently detected STBC cannot be applied in conjunction with non-coherently detected FFH. Hence, differentially encoded modulation schemes, such as differential phase shift keying (DPSK), have been used in conjunction with FFH [8], [9].

The concept of combining orthogonal transmit diversity designs with the principle of sphere packing (SP) modulation was introduced in [10]. For a simple Alamouti STBC scheme [1], it was shown in [10] that the diversity product of an orthogonal transmit diversity scheme is determined by the minimum Euclidean distance of the transmission vectors. Therefore, in order to maximize the achievable coding advantage, it was proposed to use sphere packing schemes, which have the best known minimum Euclidean distance. The results of [10] demonstrated that sphere packing aided STBCs are capable of substantially outperforming the conventional STBC [1]. Since DSTBC may be viewed as a differential encoding process followed by a STBC encoder, it is natural to apply sphere packing to DSTBC.

Iterative decoding of spectrally efficient modulation schemes was considered by several authors [11] [12]. The authors of [14] proposed a novel system that exploits the advantages of both iterative demapping and decoding [13] as well as those of the STBC-SP scheme of [10]. The STBCSP demapper of [10] was modified in [14] for the sake of 
TABLE I

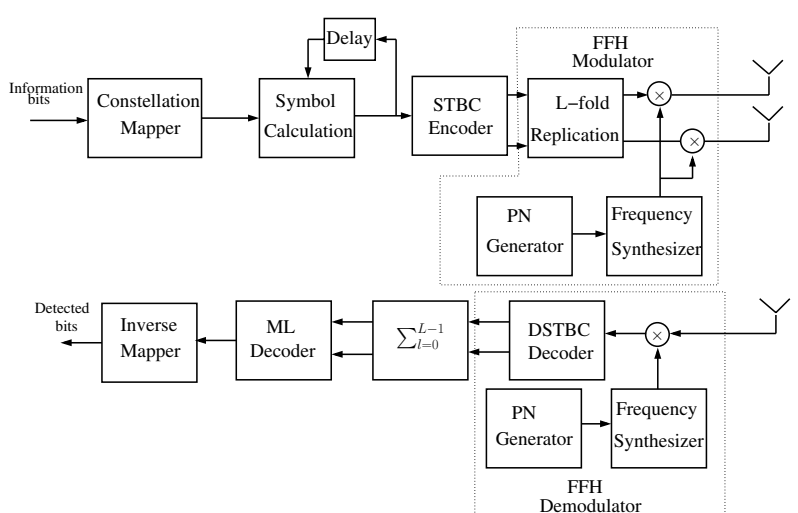

Fig. 1. Schematic of the FFH system using DSTBC and $L$-order frequency diversity

accepting the apriori information passed to it from the channel decoder as extrinsic information.

Motivated by the performance improvements reported in [13] [14] and by the benefits of achieving combined spatial and frequency diversity, in this contribution we propose a hybrid FFH assisted DSTBC scheme capable of benefitting from both the frequency diversity achievable using the FFH scheme and from the spatial diversity offered by the DSTBC arrangement, thus achieving a higher diversity order than either of the two systems in isolation. This system also benefits from the advantage of iterative soft demapping and decoding combined with sphere packing. These advantages become particularly beneficial, when the spatial or frequency diversity order is limited either as a consequence of limited antenna spacing or because the total system bandwidth is insufficient for the FFH scheme to hop beyond the channel's coherence bandwidth.

We commence our discourse by providing a detailed description of the proposed uncoded system in Section II, demonstrating how the spatial diversity and frequency diversity gains may be combined. In Section III, the channel coded system combined with sphere packing is introduced, which benefits from turbo detection. Our simulation results are discussed in Section IV. Finally, we conclude our discourse in Section V.

\section{SYSTEM DESCRIPTION FOR THE FFH-DSTBC SYSTEM}

We consider a wireless communication system invoking multiple transmit antennas at the Base Station (BS) and an arbitrary number of receive antennas at the Mobile Station (MS). The block diagram of the system amalgamating the advantages of FFH and STBC is shown in Fig.1, which employs two transmit and a single receive antenna, although the concept may be applied to an arbitrary number of receive antennas.

The DSTBC encoder was outlined in [3]. First, the information bits are mapped to the PSK constellation symbols defined by

$$
\mathcal{A}=\frac{e^{j 2 \pi n / M}}{\sqrt{2}}, \quad, n=0,1, \ldots, M-1
$$

TRANSMISSION OF SYMBOLS $x^{1}(t)$ AND $x^{2}(t)$ USING THE TRANSMIT ANTENNAS $T x_{1}$ AND $T x_{2}$ AND $L$ FFH HOPS

\begin{tabular}{|c|c|c|l|c|}
\hline & Hop 1 & Hop 2 & $\cdots$ & Hop $L$ \\
$f_{0}$ & $f_{1}$ & $\cdots$ & $f_{L-1}$ \\
\hline$T x_{1}$ & $x^{1}(t, 0)$ & $x^{1}(t, 1)$ & $\cdots$ & $x^{1}(t, L-1)$ \\
\hline$T x_{2}$ & $x^{2}(t, 0)$ & $x^{2}(t, 1)$ & $\cdots$ & $x^{2}(t, L-1)$ \\
\hline
\end{tabular}

where $x^{i}(t, l)$ denotes the replica of the $i$ th symbol transmitted at time $t$ during the $l$ th hop, $l=0,1, \ldots, L-1$ and $i=1,2$

where we have $M=2^{b}, b$ is the number of bits per symbol and $j=\sqrt{-1}$. We will restrict our attention to the binary scenario, although higher-order PSK schemes and Quadrature Amplitude Modulation (QAM) [6] may also be used. Hence, in our system we have $M=2$. Note that the division by $\sqrt{2}$ in Equation (1) is required to ensure that the total transmitted power of the two antennas becomes 1 . Let $x^{1}(2 t+1)$ and $x^{2}(2 t+1)$ denote the differentially encoded symbols during the symbol period $(2 t+1)$. These symbols are calculated based on both the symbols transmitted during the interval $(2 t-1)$ and the incoming PSK symbols denoted by $S_{i}$, where $i$ represents the transmit antenna index. This process can be expressed as [3]

$$
\begin{array}{r}
\left(x^{1}(2 t+1), x^{2}(2 t+1)\right)=\mathcal{S}_{1}\left(x^{1}(2 t-1), x^{2}(2 t-1)\right) \\
+\mathcal{S}_{2}\left(-x^{2 *}(2 t-1), x^{1 *}(2 t-1)\right) .
\end{array}
$$

Then a conventional STBC encoder is used, which obeys the $G_{2}$ transmission matrix and transmits the differential encoded symbols in time slots $(2 t+1)$ and $(2 t+2)$ according to

$G_{2}\left(x^{1}(2 t+1), x^{2}(2 t+1)\right)=\left[\begin{array}{cc}x^{1}(2 t+1) & x^{2}(2 t+1) \\ -x^{2 *}(2 t+1) & x^{1 *}(2 t+1)\end{array}\right]$.

Following the STBC encoder, the signals are modulated by the FFH modulator of Fig.1. The FFH modulator, consisting of the PN generator and the frequency synthesizer, creates $L$ chip-based replicas of the encoded symbols and transmits them in $L$ consecutive hops using a different frequency during each hop. More precisely, during each symbol duration, the FFH modulator outputs a pattern of $L$ frequencies, each of which is invoked during a hop duration of $T_{h}=T_{s} / L$, where $T_{s}$ is the symbol duration. Thus, during each hop duration, a copy of the encoded symbols is transmitted using a different frequency. This arrangement is shown for arbitrary symbols $x^{1}(t)$ and $x^{2}(t)$ in Table I, where $x^{i}(t, l)$ denotes the replica of the $i$ th symbol transmitted at time $t$ during the $l$ th hop, $l=$ $0,1, \ldots, L-1$ and $i=1,2$. Note that during any of the hops, the same frequency is used for both symbols to be transmitted using antennas 1 and 2. Moreover, the transmitted power of the symbol $x^{i}(t, l)$ is $1 / L$ times that of the original encoded symbol $x^{i}(t)$. Thus, if symbols $x^{1}(2 t+1)$ and $x^{2}(2 t+1)$ are input to the FFH modulator, then the signals transmitted during the $l$ th hop at time $(2 t+1)$ and $(2 t+2)$ may be expressed as

$$
s^{1}(2 t+1, l)=\sqrt{\frac{2 P}{L}} x^{1}(2 t+1, l) e^{j 2 \pi f_{l} t+\phi_{l}},
$$




$$
\begin{aligned}
s^{2}(2 t+1, l) & =\sqrt{\frac{2 P}{L}} x^{2}(2 t+1, l) e^{j 2 \pi f_{l} t+\phi_{l}}, \\
s^{1}(2 t+2, l) & =-\sqrt{\frac{2 P}{L}} x^{2 *}(2 t+1, l) e^{j 2 \pi f_{l} t+\phi_{l}}, \\
s^{2}(2 t+2, l) & =\sqrt{\frac{2 P}{L}} x^{1 *}(2 t+1, l) e^{j 2 \pi f_{l} t+\phi_{l}},
\end{aligned}
$$

where $P$ denotes the power of the encoded symbols $x^{1}(2 t+1)$ and $x^{2}(2 t+1)$, while $\phi_{l}$ is the phase induced by the FFH scheme. The transmitted symbols $s^{1}(2 t+1, l)$ and $s^{2}(2 t+1, l)$ satisfy the constraint of $s^{1}(2 t+1, l)^{2}+s^{2}(2 t+1, l)^{2}=1 / L$ and the total power emitted in a symbol duration is the same as that of the conventional DSTBC system, namely unity.

The channel is modeled as correlated Rayleigh fading channel for each carrier of the hopping frequency set. The rate of fading is governed by the normalized Doppler frequency given by $f_{d}=F_{d} T_{h}$, where $F_{d}$ is the Doppler frequency of $F_{d}=f_{c} v / c$, and $f_{c}$ is the carrier frequency, $v$ is the speed of the MS relative to the BS and $c$ is the speed of light. We assume that the separation between adjacent FFH carriers is at least as high as the channel's coherence bandwidth. Moreover, the spatial separation between transmit antennas in the BS is assumed to be higher than half the wavelength of the carrier frequency used. Thus, the fading experienced by all diversity channels, regardless whether constituted by different frequencies or transmit antennas, are independent.

In the receiver shown in Fig.1, the signals received by a single antenna are dehopped by the FFH demodulator. It is assumed that the FFH demodulator is perfectly frequency aligned with the FFH modulator. The dehopped received signals during the $l$ th hop at time $(2 t+1)$ are given by

$$
r(2 t+1, l)=h_{1, l} s^{1}(2 t+1, l)+h_{2, l} s^{2}(2 t+1, l)+n_{2 t+1, l},
$$

where $h_{m, l}$ denotes the path gain from the $m$ th transmit antenna to the receiver antenna during the $l$ th hop, $n_{2 t+1, l}$ is the corresponding complex Additive White Gaussian Noise (AWGN) and we have $m=1,2$ in the scenario considered. Following dehopping, the detection decision statistics $\tilde{\mathcal{S}}_{1}(l)$ and $\tilde{\mathcal{S}}_{2}(l)$ generated for the $l$ th hop of the transmitted PSK symbols using the DSTBC decoder at symbol interval $(2 t+1)$ are computed [3], as shown in Fig.1, namely

$$
\begin{aligned}
\tilde{\mathcal{S}}_{1}(l) & =r(2 t+1, l) r^{*}(2 t-1, l)+r(2 t, l) r^{*}(2 t+2, l) \\
& =\left(\left|h_{1, l}\right|^{2}+\left|h_{2, l}\right|^{2}\right) S_{1}+\tilde{N}_{1} \\
\tilde{\mathcal{S}}_{2}(l) & =r(2 t+1, l) r^{*}(2 t, l)-r(2 t-1, l) r^{*}(2 t+2, l) \\
& =\left(\left|h_{1, l}\right|^{2}+\left|h_{2, l}\right|^{2}\right) S_{2}+\tilde{N}_{2},
\end{aligned}
$$

where $\tilde{N}_{1}$ and $\tilde{N}_{2}$ are the combined noise vectors.

Once $L$ decision statistics corresponding to one symbol duration have been computed, they are summed over the $L$ corresponding hops to generate the resultant decision statistics as seen in Fig.1. We can assume that the phase $\phi_{l}$ induced by FFH, as expressed in (4), is approximately the same for a particular hopping frequency. In other words, for a particular value of $l, \phi_{l}$ is the same during all symbol intervals. Under

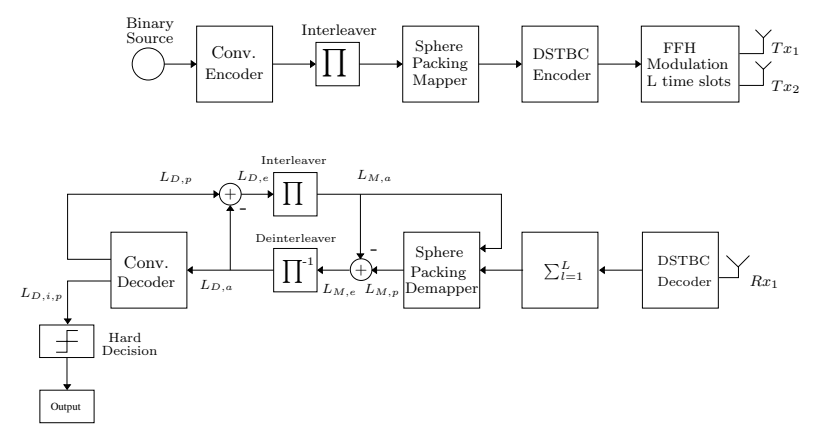

Fig. 2. Schematic of turbo detection DSTBC-SP system using $L$-order frequency diversity

the above assumption, the resultant decision statistics for a particular pair of symbols can be obtained by the summation of the decision statistics for all hops and may be expressed as

$$
\tilde{\mathcal{S}}_{1}=\sum_{l=0}^{L-1} \tilde{\mathcal{S}}_{1}(l) \quad \tilde{\mathcal{S}}_{2}=\sum_{l=0}^{L-1} \tilde{\mathcal{S}}_{2}(l) .
$$

The maximum likelihood decision is made by choosing the particular symbol that is closest to the decision statistics $\tilde{\mathcal{S}}_{1}$ or $\tilde{\mathcal{S}}_{2}$. Finally, the inverse mapping of the received symbol is invoked for the sake of decoding the transmitted bits, as shown in Fig.1.

In the context of the single receive antenna aided FFH assisted DSTBC system, the total achievable diversity order is given by $(T \times L)$, where $T$ is the number of transmit antennas and $L$ is the number of hops per symbol. Thus, without invoking a high number of transmit antennas for attaining a high spatial diversity, the proposed system is capable of achieving a high overall diversity gain.

\section{CHANNEL-CODED FFH AIDED DSTBC USING SPHERE PACKING AND ITERATIVE DECODING}

In Section II, the FFH aided DSTBC system has been discussed. In this section, sphere packing modulation and iterative decoding techniques have been integrated with the system for the sake of providing both a further coding gain and time diversity gain. Thus, the proposed system will benefit from the joint advantages of spatial, time and frequency diversity. The schematic of the entire system is shown in Fig.2.

As mentioned in Section II, two symbols, corresponding to $S_{1}$ and $S_{2}$ in the system of Fig.1, are transmitted using two time slots. Let us assume that there are $Q$ legitimate DSTBC transmitted signals $G_{2}\left(x_{q, 1}, x_{q, 2}\right), q=0,1, \cdots, Q-1$ that the encoder may choose from, which have to be differentially encoded and transmitted over two consecutive time slots. For example, in case of D-BPSK modulation and two transmit antennas we have $Q=4$ legitimate jointly designed DSTBC sphere-packed symbols. More explicitly, following the philosophy of sphere packing [15], our aim is to design $x_{q, 1}$ and $x_{q, 2}$ jointly, such that they have the best minimum Euclidean distance from all other $(Q-1)$ legitimate DSTBC transmitted 
signals, since this minimizes the system's error probability. Let $\left(a_{q, 1}, a_{q, 2}, a_{q, 3}, a_{q, 4}\right), q=0,1, \cdots, Q-1$, be the complexvalued phasor points selected from the four-dimensional realvalued Euclidean space $R^{4}$, where each of the four elements represents a specific coordinate of the jointly designed $Q=4$ DSTBC phasor points. Subsequently, $x_{q, 1}, x_{q, 2}$ maybe written as

$$
\left\{x_{q, 1}, x_{q, 2}\right\}=\left\{a_{q, 1}+j a_{q, 2}, a_{q, 3}+j a_{q, 4}\right\} .
$$

In the four-dimensional real-valued Euclidean space $R^{4}$, the lattice $D_{4}$ is defined as a sphere packing having the best minimum Euclidean distance from all other $(Q-1)$ constellation points in $R^{4}$ [15]. More specifically, $D_{4}$ may be defined as a lattice that consists of all legitimate points having integer coordinates $\left[a_{1} a_{2} a_{3} a_{4}\right]$ obeying $a_{1}+a_{2}+$ $a_{3}+a_{4}=k$, where $k$ is an even integer. Assuming that $S=\left\{\left[a_{q, 1} a_{q, 2} a_{q, 3} a_{q, 4}\right] \in R^{4}, 0 \leq q \leq Q-1\right\}$ constitutes a set of $Q$ legitimate constellation points having a total energy of $E=\sum_{q=0}^{Q-1}\left(\left|a_{q, 1}\right|^{2}+\left|a_{q, 2}\right|^{2}+\left|a_{q, 3}\right|^{2}+\left|a_{q, 1}\right|^{4}\right)$, and upon introducing the notation

$$
C_{q}=\sqrt{\frac{2 Q}{E}} G_{2}\left(x_{q, 1}, x_{q, 2}\right), q=0,1, \cdots, Q-1
$$

we have a set of vectors, whose diversity product is determined by the minimum Euclidean distance of the set of $Q$ legitimate constellation points in $S$.

As shown in Fig.2, the transmitted source bits are convolutionally encoded and then interleaved by a random bit interleaver. A rate $R=\frac{1}{2}$ Recursive Systematic Convolutional (RSC) code having a constraint length of $K=5$ and octal generator polynomials $\left(G_{r}, G\right)=(35,23)$ was employed. After channel interleaving, the sphere packing mapper of Fig. 2 maps $B$ number of channel-coded information bits $b=$ $b_{0, \cdots, B-1} \in(0,1)$ to a sphere packing modulated symbol $C_{q}$ using Equation (9). Then we partition the SP symbols into two complex-valued symbols using Equation (8), which are fed into the DSTBC encoder, followed by the FFH scheme. Hence, the latter part of the system is the same as that seen in Fig.1. However, because the pair of complex-valuedpartitioned sphere packing symbols does not have the same amplitude as the symbols of the PSK constellation, the DSTBC encoder has to invoke a differential encoding process originally proposed for QAM constellations in [6].

At the receiver seen in Fig.2, the iterative bit-based demapping and soft decoding strategy of [14] was used. The FFH demodulator was outlined in Section II and again, the DSTBC decoder of [6] designed for QAM was employed. We also note that different bit-to-phasor constellation mappings may have extremely different effects on the achievable iterative performance. In our system, the Anti-Gray Mapping (AGM) scheme of [13] was invoked for mapping the bits to sphere packing symbols.

\section{SIMULATION RESULTS AND DISCUSSION}

In this section, we first characterize the achievable performance of the uncoded FFH aided DSTBC system,

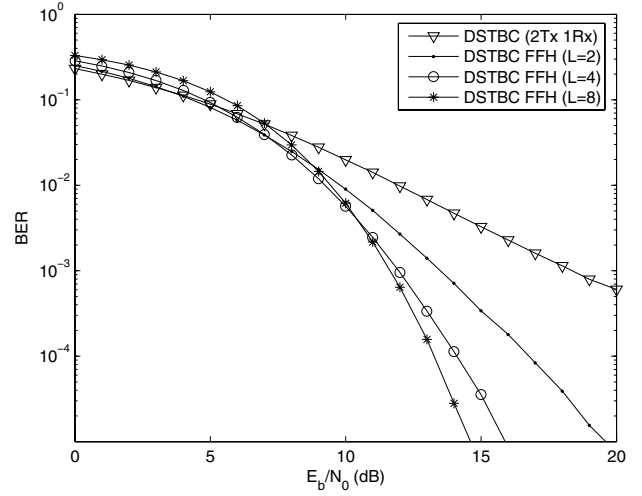

Fig. 3. BER performance of the FFH assisted DSTBC BPSK system employing two transmit and one receive antenna, when communicating over correlated Rayleigh fading channels having $f_{d}=10^{-2}$ and assuming various orders of frequency diversity $L$.

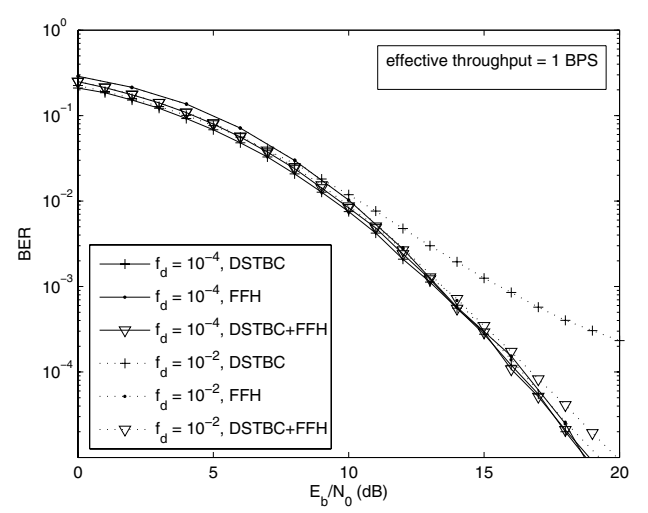

Fig. 4. BER performance comparison of the three systems having a total diversity of $D_{\text {total }}=4$ of an effective throughput of 1BPS, when communicating over correlated Rayleigh fading channels, having both $f_{d}=$ $10^{-2}$ and $10^{-4}$.

followed by that of the channel coded iterative decoding aided system. In Fig. 3, the performance of a DSTBC scheme employing two transmit and one receive antenna, assisted by a FFH arrangement using $L=2,4$ and 8 hops per symbol has been shown, when communicating over Rayleigh fading channels experiencing a relatively fast fading having a normalized Doppler frequency of $f_{d}=10^{-2}$. As seen in Fig. 3, the performance of the DSTBC system dispensing with FFH shows an error floor in the high-SNR region. This is because the differential decoding scheme ideally requires the fading to be constant for four consecutive DSTBC symbol time slots. Thus, when the fading is rapidly fluctuating, this condition cannot be satisfied and hence encountering an error floor is inevitable. However, when frequency diversity is introduced, the above-mentioned error floor can be essentially eliminated, despite the fading envelope's fluctuation experienced during the consecutive time slots.

If we fix the system's total diversity order to $D_{\text {total }}=T \times L=4$, we can have three alternative systems, namely the DSTBC $(T=4, L=1)$, the FFH $(T=1, L=4)$ and the DSTBC + FFH $(T=2, L=2)$ 
TABLE II

COMPARISON OF VARIOUS SYSTEM PARAMETERS FOR THE THREE SYSTEMS, HAVING A TOTAL DIVERSITY ORDER $D_{\text {total }}=4$

\begin{tabular}{|c|c|c|c|}
\hline \multirow{2}{*}{ Parameter } & \multicolumn{3}{|c|}{ System } \\
\cline { 2 - 4 } & DSTBC & 1 & FFH \\
\hline$T^{\vee}$ & 4 & 4 & 2 \\
\hline$L^{\Delta}$ & 1 & at $f_{d}>10^{-2}$ & 2 \\
\hline Degradation $^{*}$ & at $f_{d}>10^{-3}$ & 1 & at $f_{d}>10^{-2}$ \\
\hline Complexity $^{\boldsymbol{*}}$ & 4 & $4 A$ & 2 \\
\hline Bandwidth & $A$ & not affected & affected if antenna spacing $<\lambda / 2$ \\
\hline Spatial correlation & affected if antenna spacing $<\lambda / 2$ & affected if frequency spacing $<B_{c}$ & affected if frequency spacing $<B_{c}$ \\
\hline Frequency correlation & not affected &
\end{tabular}

$\nabla \mathrm{T}$ is the number of transmit antennas. ${ }^{\Delta} \mathrm{L}$ is number of hops per symbol. ${ }^{*}$ Degradation is in BER when compared to performance at $f_{d}=10^{-4}$. ${ }^{*}$ Complexity is measured by the number of transmit antennas. ${ }^{\circ} A$ denotes the bandwidth of the narrowband DSTBC system. $B_{c}$ is the channel coherence bandwidth.

arrangements, corresponding to different combinations of spatial and frequency diversity. Fig. 4 explicitly demonstrates that for slow fading having a normalized Doppler frequency of $f_{d}=10^{-4}$ all the systems exhibit a similar performance. However, when the fading becomes moderately fast, such as $f_{d}=10^{-2}$, the DSTBC system, which requires the fading envelope to be constant for eight consecutive time slots, degraded more significantly than the others. Therefore, we may conclude that the FFH assisted DSTBC system is capable of supporting higher vehicular speeds than the pure DSTBC system having the same diversity order, when considering a fixed transmission rate. A more detailed comparison of the three systems can be found in Table II.

For the convolutionally coded FFH aided DSTBC scheme using sphere packing modulation, the following parameters have been used for generating the results. The transmission frame length was 1000 information bits and the interleaver's length was set to 2000. The highest iteration gain was achieved using Anti-Gray Mapping [13] by the turbo-detected sphere packing scheme. Since a half-rate RSC encoder was used and the DSTBC scheme transmitted one sphere packing symbol during two consecutive time slots, the effective throughput of the 16-point half-rate coded sphere packing scheme was $R_{\text {bps }}=0.5 \times 4 \times 0.5=1$. The channel was assumed to have a Doppler fading rate of $f_{d}=10^{-2}$.

Fig. 5 portrays the attainable system performance both with and without sphere packing. For the sake of a fair comparison, QPSK modulation having an effective throughput of 1 BPS and using set partitioning was invoked for the system dispensing with sphere packing. The non-iterative sphere packed system does not show any advantage, but when the number of iterations is increased, the system will have an approximately $0.8 d B$ iteration gain at BER $=10^{-5}$ in conjunction with an interleaver length of 2000. This gain is achieved, because sphere packing is jointly designed for two consecutive DSTBC symbols. Therefore, when decoding a single symbol, we will be able to glean information from all the four bits of a symbol. When the interleaver's length (IL) is increased from $I L=2000$ to $I L=20000$ bits, the gain due to sphere packing is increased to $1.5 \mathrm{~dB}$.

The attainable iteration gain as well as the FFH-induced

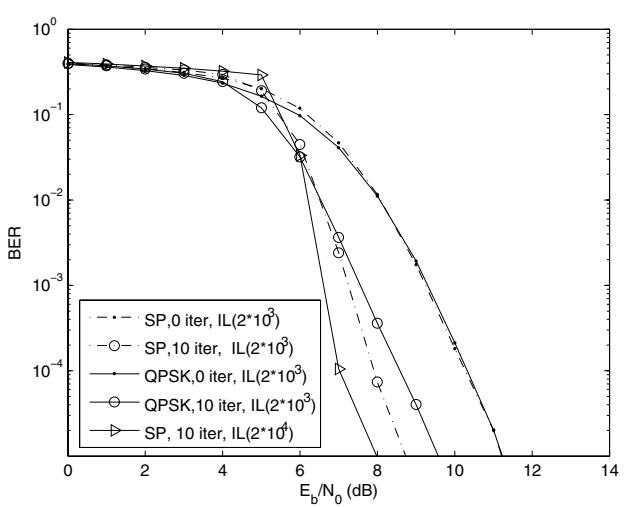

Fig. 5. BER performance comparison of the half-rate RSC-coded system both with/without Sphere Packing(SP), using open loop and 10 iterations (iter) and an interleaver length (IL) of IL $=2000$ and 20000 bits, when communicating over correlated Rayleigh fading channels, having $f_{d}=10^{-2}$, and FFH $(\mathrm{L}=2)$.

frequency diversity gain are shown in Fig. 6. When comparing $\mathrm{FFH}$ using both $\mathrm{L}=4$ and $\mathrm{L}=1$, the system attains an approximately $1.6 d B$ frequency diversity gain, regardless of the number of iterations used. When comparing the scenarios invoking 10 iterations and no iteration along with FFH having $L=2$ hops, the systems characterized in Fig. 6 exhibit an approximately $2.6 d B$ iteration gain. In fact, when using as few as four iterations, the system will have achieved an approximately $2.4 d B$ iteration gain.

In Fig. 7, we characterize the achievable system performance at different fading rates. Channel coding has the potential of spreading the effect of an information bit across numerous consecutive encoded bits and hence the system may be deemed to have time diversity. Our half-rate RSC-coded system now exhibits combined time, spatial as well as frequency diversity. Time diversity requires the fading envelope of adjacent bits to be independent. By contrast, the nature of DSTBC requires the fading envelope to be constant during a number of consecutive symbols for the sake of achieving a high spatial diversity gain. Therefore, it depends on the fading rate encountered, whether time diversity or spatial diversity or both are achieved. The best overall system performance was achieved at $f_{d}=10^{-2}$, corresponding to the best spatial versus time diversity balance. When the fading 


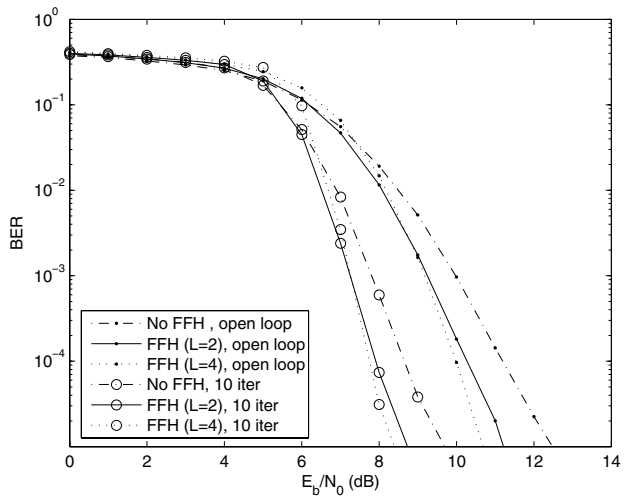

Fig. 6. BER performance of half-rate RSC-coded system using various orders of frequency diversity $L$ and various number of iterations (iter), when communicating over correlated Rayleigh fading channels, having $f_{d}=10^{-2}$.

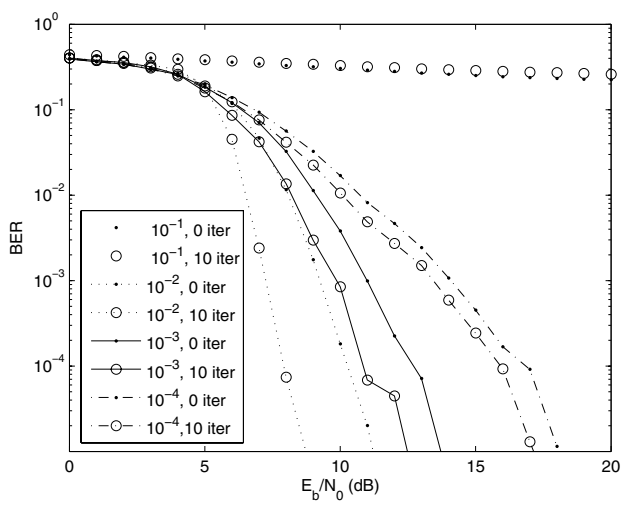

Fig. 7. BER performance of the coded system, when communicating over different fading rate $\left(f_{d}=10^{-1}, 10^{-2}, 10^{-3}, 10^{-4}\right)$ correlated Rayleigh channels, having 0,10 decoding iterations(iter) and $\mathrm{FFH}(\mathrm{L}=2)$.

rate becomes lower, such as $f_{d}=10^{-3}$ or $10^{-4}$, the system becomes capable of benefitting from a higher spatial diversity, while the benefits of time diversity will be gradually eroded. On the other hand, under fast fading conditions associated with $f_{d}=10^{-1}$, DSTBC becomes incapable of attaining a high spatial diversity gain, while the achievable time diversity gain becomes significant. The highest iteration gain can be achieved at $f_{d}=10^{-2}$.

\section{CONCLUSION}

We have proposed a sophisticated non-coherently detected iterative decoding aided system that achieves an increased spatial and frequency diversity by combining DSTBC with FFH. We have shown using simulation results that the proposed system having $L$-th order frequency diversity and two transmit antennas approaches the BER performance of a pure DSTBC system employing $(L \times 2)$ number of transmit antennas. However, the advantage of the proposed system is that it is less vulnerable to the increase of the fading rate than DSTBC. When compared to a pure FFH based DPSK system dispensing with spatial diversity, the proposed FFH based
DSTBC arrangement is capable of achieving a higher diversity order at a reduced bandwidth requirement. Furthermore, by increasing the number of frequency hops per symbol, a higher diversity gain is achievable using the proposed FFH based DSTBC scheme, when compared to the conventional DSTBC system. In conclusion, the advantages of DSTBC, FFH, sphere packing and iterative decoding may be jointly capitalized on by the proposed system, hence offering more advanced features and a better performance than any of its components, which is achieved without having to estimate the channel. Our future research will explore the potential of sophisticated mapping and precoding techniques with the aid of EXIT-chart analysis.

\section{REFERENCES}

[1] S. M. Alamouti, "A simple transmit diversity technique for wireless communications," IEEE Journal on Selected Areas of Communications, vol. 16, pp. 1451-1458, October 1998.

[2] V. Tarokh, H. Jafarkhani, and A. R. Calderbank, "Space-time block codes from orthogonal designs," IEEE Transactions on Information Theory, vol. 45, pp. 1456 - 1467, July 1999.

[3] V. Tarokh and H. Jafarkhani, "A differential detection scheme for transmit diversity," IEEE Journal on Selected Areas of Communications, vol. 18, pp. 1169 - 1174, July 2000.

[4] B. M. Hochwald and W. Sweldens, "Differential unitary space-time modulation," Communications, IEEE Transactions on, vol. 48, no. 12, p. $2041,2000$.

[5] B. L. Hughes, "Differential space-time modulation," Information Theory, IEEE Transactions on, vol. 46, no. 7, p. 2567, 2000.

[6] C. Hwang, S. Nam, J. Chung, and V. Tarokh, "Differential space time block codes using nonconstant modulus constellations," Signal Processing, IEEE Transactions on [see also Acoustics, Speech, and Signal Processing, IEEE Transactions on], vol. 51, no. 11, p. 2955, 2003. Differential space time block codes using nonconstant modulus constellations.

[7] R. E. Ziemer and R. L. Peterson, Digital communications and spread spectrum systems. New York: Mcmillan Inc., 1985.

[8] R. W. Nettleton and G. R. Copper, "Performance of a frequencyhopped differentially modulated spread-spectrum receiver in a Rayleigh fading channel," IEEE Transactions on Vehicular Technology, vol. VT30, pp. $14-29$, February 1981.

[9] G. R. Cooper and R. W. Nettleton, "A spread-spectrum technique for high-capacity mobile communications," IEEE Transactions on Vehicular Technology, vol. VT-27, pp. 264 - 275, Novemebr 1978.

[10] W. Su, Z. Safar, and K. J. R. Liu, "Space-time signal design for timecorrelated rayleigh fading channels," Communications, 2003. ICC '03. IEEE International Conference on, vol. 5, p. 3175, 2003.

[11] S. L. Goff, A. Glavieux, and C. Berrou, "Turbo-codes and high spectral efficiency modulation," Proceedings of IEEE International Conference on Communications, pp. 645-649, May 1994.

[12] T. Mittelholzer, X. Lin, and J. Massey, "Multi-level turbo coding for $\mathrm{m}$-ary quadrature and amplitude modulation," International Symposium on Turbo Codes and Related Topics, pp. 127 - 134, Sep 1997.

[13] S. ten Brink, J. Speidel, and R.-H. Yan;, "Iterative demapping and decoding for multilevel modulation," Global Telecommunications Conference, GLOBECOM 98. The Bridge to Global Integration. IEEE, vol. 1, pp. 579 - 584, 1998.

[14] O. Alamri, B. L. Yeap, and L. Hanzo, "Turbo detection of channelcoded space-time signals using sphere packing modulation," Vehicular Technology Conference, 2004. VTC2004-Fall. 2004 IEEE 60th, vol. 4, pp. 2498-2502, 2004.

[15] J. Conway and N. Sloane, Sphere Packing, Lattices and Groups. Springer-Verlag, 1999. 